\title{
Looking on the bright side: The story of AA Doradus as revealed by its cool companion $\star$
}

\author{
M. Vučković ${ }^{1,5}$, R. H. Østensen ${ }^{2}$, P. Németh ${ }^{2,3}$, S. Bloemen ${ }^{2,4}$, and P. I. Pápics ${ }^{2}$ \\ 1 Instituto de Física y Astronomía, Facultad de Ciencias, Universidad de Valparaíso, Gran Bretaña 1111, Playa Ancha, \\ 2360102 Valparaíso, Chile \\ e-mail:maja.vuckovic@uv.cl \\ 2 Instituut voor Sterrenkunde, KU Leuven, Celestijnenlaan 200D, 3001 Leuven, Belgium \\ 3 Dr. Karl-Remeis-Observatory \& ECAP, Astronomical Institute, F.-A.-U. Erlangen-Nürnberg, 96049 Bamberg, Germany \\ 4 Department of Astrophysics, IMAPP, Radboud University Nijmegen, PO Box 9010, 6500 GL Nijmegen, The Netherlands \\ 5 Astronomical Observatory of Belgrade, Volgina 7, 11060 Belgrade, Serbia
}

Received 18 May 2015 / Accepted 1 October 2015

\section{ABSTRACT}

\begin{abstract}
The effects of irradiation on the secondary stars of close binary systems are crucial for reliably determining the system parameters and for understanding the close binary evolution. They affect the stellar structure of the irradiated star and are reflected in the appearance of characteristic features in the spectroscopic and photometric data of these systems. We aim to study the light that originates from the irradiated side of the low-mass component of a close binary eclipsing system, which comprises a hot subdwarf primary and a low mass companion, to precisely interpret their high precision photometric and spectroscopic data, and accurately determine their system and surface parameters. We reanalyse the archival high-resolution time-resolved VLT/UVES spectra of AA Dor system, where irradiation features have already been detected. After removing the predominant contribution of the hot subdwarf primary, the residual spectra reveal more than 100 emission lines from the heated side of the secondary, which show maximum intensity close to the phases around the secondary eclipse. We analyse the residual spectrum to model the irradiation of the low-mass secondary. We perform a detailed analysis of 22 narrow emission lines of the irradiated secondary, mainly of O II, with a few significant C II lines. Their phase profiles constrain the emission region of the heated side to a radius $\geq 95 \%$ of the radius of the secondary, while the shape of their velocity profiles reveals two distinct asymmetry features, one at the quadrature and the other at the secondary eclipse. In addition, we identify weaker emission signatures originating from more than 70 lines, including lines from He I, N II, Si III, Ca II, and Mg II. From the emission lines of the heated side of the secondary star, we determine the radial velocity semi-amplitude of the centre-of-light and correct it to the centre-of-mass of the secondary which, in turn, gives accurate masses of both components of the AA Dor system. The resulting masses $M_{1}=0.46 \pm 0.01 M_{\odot}$ and $M_{2}=0.079 \pm 0.002 M_{\odot}$ are in perfect accordance with those of a canonical hot subdwarf primary and a low mass that is just at the substellar limit for the companion. We also compute a first generation atmosphere model of the low mass secondary, which includes irradiation effects and matches the observed spectrum well. We find an indication of an extended atmosphere of the irradiated secondary star.
\end{abstract}

Key words. binaries: eclipsing - subdwarfs - stars: low-mass - stars: general

\section{Introduction}

The booming research field of hot-subdwarf stars has been enriching our knowledge on very broad and diverse fields of astrophysics, all the way from stellar evolution and asteroseismology to planet findings (see Heber 2009, for a review). However, these unique stellar laboratories offer further opportunities. As a significant fraction of hot subdwarfs are found in binaries (Maxted et al. 2001; Napiwotzki et al. 2004; Morales-Rueda et al. 2006; Copperwheat et al. 2011), they present a superb stellar population to study binary evolution. In fact, the leading formation channels for these stars involve, in one way or another, binary evolution processes (Han et al. 2002, 2003).

More than 100 binary hot subdwarfs have been found in short-period systems with periods ranging from a few hours to several days, with companions being either white-dwarf (WD) or a low mass M-dwarf stars (dM) (Kawka et al. 2015). Such short-period systems must have formed through binary mass transfer, followed by common-envelope ejection (CEE). Due

$\star$ Based on observations collected at the European Southern Observatory, Chile. Program ID: 066.D-1800. to their extremely thin $\left(<0.02 M_{\odot}\right)$, and hence inert hydrogen envelope, the subdwarfs evolve directly into WDs, bypassing the asymptotic giant branch (AGB) and planetary nebula (PN) phases (Heber et al. 1984; Saffer et al. 1994). Because the shortest period hot subdwarf $+\mathrm{dM}$ systems will evolve into cataclysmic variables $(\mathrm{CVs})$ in less than a Hubble time, they are important for understanding the pre-CV evolution, with an observational advantage over the CV systems, since their binary components are not hidden in the glare of an accretion disk. Hence, the short-period hot subdwarf binaries represent an important progenitor channel of CVs. In fact, Schenker (2005) postulates that the CVs below the period gap are the product of post-subdwarf binary evolution. On the other hand, the hot subdwarfs with massive WD companions are potential progenitors of Type Ia supernovae (see Iben \& Tutukov 1984; Webbink 1984, and for the most recent candidates see Geier et al. 2013; Vennes et al. 2012). Therefore, they are an important ingredient in understanding Type Ia supernovae.

Establishing the accurate masses of both components of post-common-envelope binaries (PCEBs) is the only empirical way to constrain the efficiency parameters of the CEE phase. 
Furthermore, detailed investigation of hot subdwarf binaries is crucial when determining their masses for comparison with their theoretically proposed evolutionary channels, as well as to test the binary-population synthesis models. However, most of the hot subdwarf binary systems with known orbital periods are single-lined spectroscopic binaries, making it impossible to reliably determine the absolute masses of their components. Among the PCEBs, 15 eclipsing binary systems have been found to date, which are comprised of a hot subdwarf primary ( $\mathrm{sdO}$ or sdB star) and a low mass secondary, either a late $\mathrm{dM}$ or a brown dwarf (BD) (for the most recent discoveries see Schaffenroth et al. 2013; Barlow et al. 2013; Schaffenroth et al. 2014, 2015). Such eclipsing systems are known as HW-Vir-type binaries after the prototype. In addition to eclipses, HW-Vir systems are notorious for having a very strong "reflection effect". This reflection effect, characteristic for all close binaries that comprise a very hot primary and much cooler secondary, is due to the high contrast in the temperatures between the heated and unheated hemisphere of the secondary star. As the secondaries in these systems are supposed to be orbiting synchronously, one side of the cool secondary star is constantly being irradiated by the strong ultraviolet (UV) flux of the hot-subdwarf primary. The light-curve solutions of HW-Vir-type systems imply that the temperature of the cool companion around the substellar point is increased up to 10000-20000 K (Hilditch et al. 2003; Kiss et al. 2000). However, the detection of spectral features from the irradiated secondary in HW-Vir-type systems is hampered by the contamination from the much hotter subdwarf primary.

Recently, the eclipsing PCEBs gained even more attention, because nearly all systems with accurate eclipse-timing measurements, which have long enough data sets, are found to show apparent orbital-period changes (Kilkenny 2014) that might indicate the presence of circumbinary planets (see Beuermann et al. 2010, 2012a,b, for the most convincing cases). The claims of circumbinary planets in short-period $\mathrm{sdB}+\mathrm{dM} / \mathrm{BD}$ systems, as well as various planets in close orbits around single subdwarf B stars (Silvotti et al. 2007; Charpinet et al. 2011), show that planets may not only survive through the CE evolution, but also that they might play an important role in the formation of hot subdwarf stars (Bear \& Soker 2011; Soker 1998). Whether a planet in a PCEB system has survived the CEE or has been formed out of the ejected envelope (so-called second-generation planet, see Zorotovic \& Schreiber 2013) remains a question that current observations are just beginning to tackle. The nature of such substellar objects can be addressed through the analysis of their irradiated light, which in turn gives their masses.

Interestingly, Kilkenny (2014) showed that AA Dor has a stable orbital period to the level of $10^{-14} \mathrm{~d}$ per orbit, based on 33 years of primary eclipse timings, which was recently confirmed by analysing multi-year observations from the SuperWASP archive (Lohr et al. 2014). This makes AA Dor a unique eclipsing subdwarf PCEB system as all other HW-Vir-type systems measured with sufficient accuracy show semi-periodic variations in the eclipse timings.

In this paper we present a detailed study of the strong irradiation effect in AA Dor, a bona fide member of the HW-Vir-type systems, in which the irradiated light from the super-heated face of the secondary was first detected by Vučković et al. (2008), and recently confirmed by Hoyer et al. (2015).

\section{The tale of AA Dor}

Ever since Kilkenny \& Hill (1975) first reported the variations in the light curve of AA Dor (also known as LB 3459), a blue star in the foreground of the Large Magellanic Cloud has never ceased to draw the attention. Even though the AA Dor system is one of the most studied short-orbit subdwarf systems, the controversy over the nature of its components still remains. The discussion on the evolutionary status of AADor started soon after the discovery with the works of Paczynski \& Dearborn (1980) and Kudritzki et al. (1982), who more or less agreed on the two most likely possibilities: that AA Dor is either a helium WD with a mass of about $0.3 M_{\odot}$ or a carbon-oxygen WD with a mass of $0.55 M_{\odot}$ with a degenerate red-dwarf companion with a mass of $0.04 M_{\odot}$ and $0.07 M_{\odot}$ respectively. However, these early speculations were disputed by Kilkenny et al. (1981) and Hilditch \& Kilkenny (1980), who proposed a new evolutionary model with a mass ratio near 0.1 and a primary of $0.5 M_{\odot}$ with a $0.07 M_{\odot}$ secondary, concluding that the primary is a subdwarf $\mathrm{O}$ star.

Determined to reveal the true nature of its companion, the first high-resolution $(R=46890)$ time-resolved spectra of AA Dor were collected by Rauch \& Werner (2003). The data set reveals the expected Rossiter-McLaughlin (RM) effect (Rossiter 1924), but it was not used to refine the parameters.

The strong reflection effect seen in the light curve implies that the secondary component is strongly heated, however, many attempts to detect spectral features of the secondary star in AA Dor failed, e.g. Hilditch et al. (1996), Rauch \& Werner (2003). This inevitably makes it impossible to derive the absolute sizes of stars in this system, and so the speculation of the masses of the stars in AA Dor continued. In a single-lined binary system, only the mass function can be determined and one is forced to make sound assumptions on the mass of one star to determine the absolute sizes of both stars. As the derived surface gravity and effective temperature of the primary fall into the extended horizontal branch (EHB) region, where the hot subdwarf stars reside, the sound assumption would be that the primary mass is the canonical mass of a hot subdwarf star. This was done by Hilditch et al. (2003), who imposed the $0.5 M_{\odot}$ for the hot subdwarf primary star and from there derived a mass of the secondary of $0.09 M_{\odot}$, which agrees with an M-dwarf with a surface temperature of about $2000 \mathrm{~K}$. However, there is another group of stars that crosses the EHB region of the $T_{\text {eff }}$ - $\log g$ diagram on their way to the WD graveyard: the postred giant branch (RGB) stars. These stars were formed by extreme mass loss on the RGB before they could ignite helium. Because of this extreme mass loss, they cannot reach the density required for the core helium flash and evolve directly into lowmass WDs (Driebe et al. 1998). By comparing the location of AA Dor, as derived from their high-resolution optical and UV spectra with the evolutionary tracks for post-RGB stars, Rauch $\&$ Werner (2003) assumed a mass of $0.33 M_{\odot}$ for the AADor primary, which consequently gave a correspondingly lower mass for the secondary component of $0.066 M_{\odot}$ - below the substellar limit (Baraffe et al. 2015).

Since AA Dor is one of the few systems where it is possible to determine its mass accurately, these numbers are used actively to constrain evolutionary models (Zorotovic et al. 2010). With the aim of searching for signatures of the irradiated secondary star, which would in turn provide us with the accurate masses of the AA Dor system, Vučković et al. (2008) reanalyse the VLT/UVES data set of Rauch \& Werner (2003), discovering more than 20 emission lines that originate from the heated side of the secondary. Based on a lower limit of the radial velocity of the irradiated object, Vučković et al. (2008) made a first estimate of the masses of AA Dor components, which were consistent with a regular EHB primary and a low-mass M-dwarf secondary, contrary to the results of Rauch \& Werner (2003). 
Refining the non-LTE models and including the proper treatment of the irradiation, Klepp \& Rauch (2011) reanalysed their VLT/UVES data set and found a full agreement with the photometric model of Hilditch et al. (2003). This solved, once and for all, the long-lasting $\log g$ problem. The mass they found for the primary, by comparing their spectroscopic solution to the evolutionary tracks of Dorman et al. (1993), rules out the post-RGB scenario and is in full accordance with the one found by Vučković et al. (2008). They derived the secondary mass of $0.079 \pm 0.007 M_{\odot}$, which once again confirmed that the secondary is at the hydrogen burning mass limit, i.e. either a brown dwarf or a late M-dwarf star.

While we were finalising this paper, one more article was published on the same star. Benefiting from fresh X-Shooter phase-dependent spectroscopy, Hoyer et al. (2015) combine their FUSE (Fleig et al. 2008) and UVES (Rauch \& Werner 2003) data to yet again search for the signatures of the low-mass secondary in the AA Dor system. They identified 57 emission lines of the secondary star in the UVES spectra and 53 spectral lines in the X-Shooter spectra. Out of these, they used 22 lines (14 from UVES data, 7 from X-Shooter UVB arm, and 1 from XShooter VIS arm) to measure the orbital centre-of-light velocity of the secondary star. Assuming that the centre of gravity of the measured irradiated light is dominated by a region lying towards the primary star, they estimated the radial velocity semi-amplitude of the secondary to be $232.9_{-6.5}^{16.6} \mathrm{~km} \mathrm{~s}^{-1}$ and correspondingly derive the masses of the both stars in AA Dor $M_{1}=0.475_{-0.0357}^{0.0975} M_{\odot}$ and $M_{2}=0.0811_{-0.102}^{0.0184} M_{\odot}$, which are in full agreement with their previous result (Klepp \& Rauch 2011), as well as with the results of Vučković et al. (2008).

\section{Wiggles and wriggles in the velocities}

While examining the orbit-subtracted radial-velocity (RV) residuals in the UVES spectra of NY Vir, Vučković et al. (2007) noticed an apparent distortion at orbital phases around the secondary eclipse. As this feature occurs right where the contribution from the irradiated side of the secondary star is at its maximum, it could therefore be an artefact of the reflected light or a fingerprint of the heated face of the secondary. Because of the complexities caused by the pulsating primary of the NY Vir system and the low $\mathrm{S} / \mathrm{N}$ of this particular data set, Vučković et al. (2007) were not able to analyse this effect any further. Motivated by this intriguing finding we then decided to search for spectra of a similar system in which a similar feature could emerge. The high S/N time-resolved UVES spectra of AA Dor obtained by Rauch \& Werner (2003) were ideal for this purpose. We retrieved this data set from the ESO archive and reduced them using the standard ESO $\mathrm{CPL}^{1}$ routines. While the individual spectra are of good quality with a typical $\mathrm{S} / \mathrm{N}$ of the order of 50 per wavelength bin, the known problem with the imperfect echelle order merging ${ }^{2}$ causes ripples in the wings of Balmer lines and the continuum level. As this is potentially fatal for our analysis and there was no way around it, we devoted special attention to this when normalising the spectra.

We performed a half-automatised, two step normalisation of each spectrum, following the procedure of Pápics et al. (2012).

\footnotetext{
CPL stands for Common Pipeline Library.

2 For a proper merging of the adjacent echelle orders, the blaze function has to be corrected. Unfortunately, in the standard UVES pipeline reduction, the blaze function is estimated from a flat-field exposure, which is obviously not capable of properly correcting the blaze function.
}

All spectra were brought to the same flux level by dividing them with their median intensity and we defined a master function (using the first spectrum) with cubic splines, which was fitted to the continuum at fixed wavelength points that were known to be free of spectral lines. We then used this function to clean all spectra from large-scale artificial features, e.g. the wiggles in the continuum caused by imperfect flat-fielding. The careful selection of these nodal points enabled us to construct a function which does not distort the shape (and especially the wings) of the Balmer lines. To correct for small-scale effects and any remaining global trend of an instrumental origin, we carried out a second normalisation, using many more nodal points that were connected by linears at this phase. The wavelengths of these points were the same for all spectra, but the normalising function was constructed for each of them based on the local flux levels, instead of applying a master function again. Using linears at this step enabled us to leave the shape of wide features - like the Balmer-wings - untouched (by placing only one nodal point at the beginning and the end of such regions), but still make small-scale corrections (by using a denser nodal point distribution where it was needed). The described process is much faster, less subjective, and provides better consistency than a one-by-one manual normalisation.

The sdO primary of the AA Dor system has a richer spectrum compared with the spectrum of the sdB primary in the NY Vir system. In addition to the broad Balmer absorption lines, the sdO star displays a strong He II line, as well as several clear metal lines, which we used to measure the RV of the primary at different orbital phases. We calculated the RVs by fitting a single Gaussian to each of the He and metal lines, while multiGaussians were fitted to the broad Balmer lines, following the procedure explained in Morales-Rueda et al. (2003). The depth of the He II line and the Balmer lines were kept as free parameters in our fits, while the depth of the metal lines were fixed at their average value. In this way we obtained the RVs of the Balmer lines from $\mathrm{H}_{\beta}$ to $\mathrm{H}_{\eta}$, of the He II line at $4685.79 \AA$ and of several metal lines, like O III at $3759.87 \AA$, Si IV at $4088.86 \AA$, and $\mathrm{Mg}$ II at $4481.23 \AA$. To each of these sets of RV measurements we fitted a sine function locked in phase to the well-known ephemeris of the system (Kilkenny 2014), leaving only the RV amplitude, $K_{\mathrm{sdO}}$, and the systemic velocity, $\gamma$, as free parameters. The results from these sine fits to the three sets of RV measurements are given in Table 1 . The residual RVs that remain, after subtracting the respective sine functions, are plotted in Fig. 1 as a function of orbital phase, $\varphi$, where the zero phase is defined as corresponding to the primary eclipse. In addition to the well known Rossiter-McLaughlin (RM) effect, first reported in these spectra by Rauch \& Werner (2003), we clearly see a broad distortion feature appearing in the Balmer lines just around the secondary eclipse $(\varphi=0.5)$, whereas it is not present in any of the metal lines, nor in the He II line. The fact that it appears only in the Balmer lines and not in the He II, nor in the metal lines, of the $\mathrm{sdO}$ star is a clear indication that this distortion is caused by the phase-dependent contamination of the Balmer lines by the corresponding lines present in the light from the irradiated hemisphere of the secondary star.

\section{Signatures from the hot side}

After carefully reprocessing and normalising the UVES spectra, as described in the previous section, we proceeded by computing a mean spectrum of the primary taken from phases close to the primary eclipse, but avoiding those affected by the eclipse. 


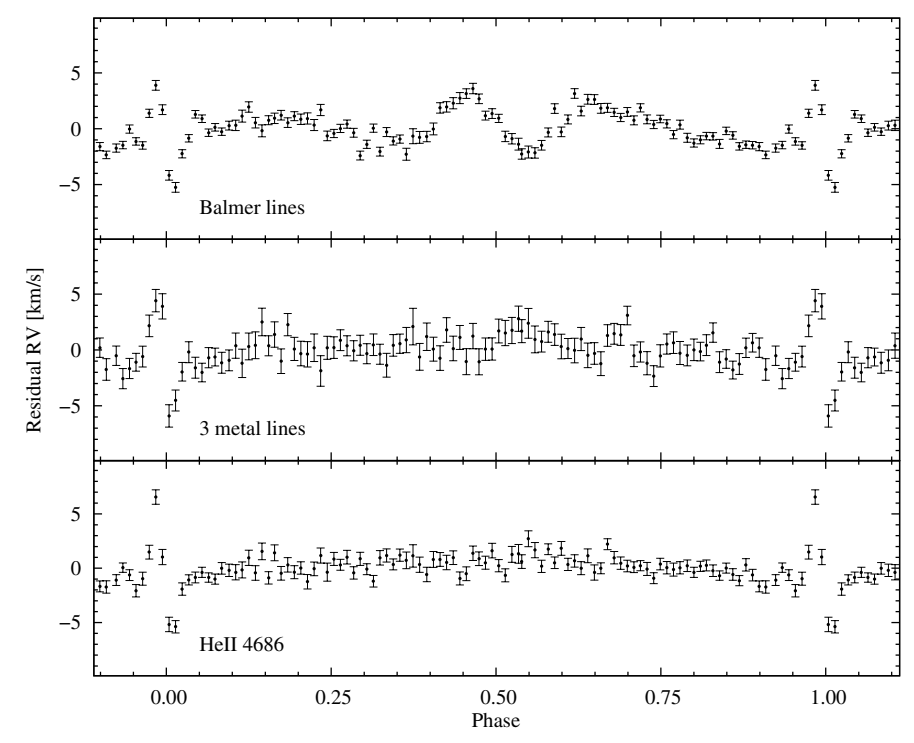

Fig. 1. The orbit subtracted RV residuals of the primary with their corresponding errors. Top panel: is for the 3 Balmer lines $\mathrm{H}_{\delta}, \mathrm{H}_{\gamma}$ and $\mathrm{H}_{\beta}$ lines. Middle panel: is for the metal lines O III at $3759.87 \AA$, Si IV at $4088.86 \AA$ and $4116 \AA$ and Mg II at $4481.23 \AA$ and the bottom panel for HeII at $4685.79 \AA$.

Table 1. Fits to the radial velocity measurements.

\begin{tabular}{lcc}
\hline \hline Spectrum & $\begin{array}{c}K_{\text {sdO }} \\
{\left[\mathrm{km} \mathrm{s}^{-1}\right]}\end{array}$ & $\begin{array}{c}\gamma \\
{\left[\mathrm{km} \mathrm{s}^{-1}\right]}\end{array}$ \\
\hline Balmer lines & $-41.81 \pm 0.22$ & $-0.88 \pm 0.16$ \\
He II 4686 & $-39.64 \pm 0.18$ & $+1.00 \pm 0.15$ \\
3 metal lines & $-39.63 \pm 0.21$ & $+0.97 \pm 0.13$ \\
\hline Adopted & $-39.63 \pm 0.21$ & $+0.97 \pm 0.13$ \\
\hline
\end{tabular}

The mean spectrum was constructed from nine individual spectra, which occurr at phases, $\varphi=0.03-0.08$, just before and after the primary eclipse when the cold side of the secondary is facing us. The spectra were shifted by

$v_{\mathrm{sdO}}=K_{\mathrm{sdO}} \sin (2 \pi \varphi)$

before averaging. The upper-right panel of Fig. 2 shows the mean spectrum around the $\mathrm{H} \gamma$ line. Each of the 105 individual spectra were then cleaned from the contribution of the sdO primary by subtracting the mean spectrum shifted according to Eq. (1). The residual spectra around the $\mathrm{H} \gamma$ line are shown in the lower-left panel of Fig. 2. The trailed residuals clearly show a very fast moving profile that changes velocity in antiphase with the primary and has a maximum intensity just before and after $\varphi=0.5$ (secondary eclipse). Superimposed on the red wing of the $\mathrm{H} \gamma$ profile, there are a number of sharp emission features that all can be identified with $\mathrm{O}$ II lines. Shifting by a velocity of $K_{\text {irr }} \approx 222 \mathrm{~km} \mathrm{~s}^{-1}$ (see Sect. 4.1) and summing the contribution between $\varphi=0.23-0.47$ produces the spectrum shown in the upper-left panel of Fig. 2. The lower-right panel of Fig. 2 shows the phase profile of this line, which is the total flux per phase bin in a $10 \AA$ window, centred on the emission profile of each spectrum.

The full emission-line spectrum of the secondary component is given in Fig. 3. All the Balmer lines from $\mathrm{H} \beta$ to $\mathrm{H} \theta$ are clearly visible and indicated by labeled arrows in the figure. $\mathrm{H} \beta$ to $\mathrm{H} \zeta$ appear to have profiles with reversed cores. As
Barman et al. (2004) explains, these reversed core profiles are produced by departures from local thermodynamic equilibrium (LTE) in layers above where the underlying emission line forms.

In Table A. 1 we provide a list of all the metal lines that originate from the irradiated face of the secondary component that we could identify in the emission line spectrum. We list peak intensity and equivalent width for each of the 22 metal lines that we have retained for use in the further analysis.

\subsection{Emission line profiles}

The phase profile of the emission lines is essentially the light curve of the irradiated star. As can be seen from the lower-right panel of Fig. 2, it shows a total eclipse at $\varphi=0.5$ and has a minimum that coincides with the primary eclipse.

Phase profiles for the strongest Balmer lines (computed as for $\mathrm{H} \gamma$ in the lower-right panel of Fig. 2) are shown in Fig. 4, as well as for the sum of all seven lines in the upper-right panel. The shape of the phase profiles provides information on the size of the emission region on the heated hemisphere of the secondary. There are no significant differences between the different Balmer lines, so we use the sum of the seven lines to analyse the phase dependence of the light from the irradiated side of the companion. It can be seen that the hot spot must cover almost the entire hemisphere of the secondary since it only reaches zero intensity immediately before and after the primary eclipse $(\varphi=0)$. To quantify this effect and determine the relationship between the radial velocity measured from the irradiated hemisphere, the centre-of-light velocity, $K_{\text {irr }}$, and the velocity of the centre of mass of the secondary, $K_{2}$, we must compute some simple geometrical models. In Sect. 4.2 below we compare the integrated Balmer-line profile with these models. While the Balmer-line profiles are very broad in velocity space, the metal line profiles are sharp enough to resolve the rotational profile of the irradiated star in velocity. We summed up the metal line profiles as we did for the Balmer lines. They show the same profiles in phase space, but with a much less signal than the Balmer-line profiles. In velocity space, however, they reveal some even more interesting features.

The sharpness of the metal-line profiles makes them well suited for determining the centre-of-light velocity, $K_{\text {irr }}$. To enhance the signal as much as possible, we used the theoretical central wavelengths of the 22 strongest lines (see Table A.1) and added them up in velocity space. The resulting velocity/phase image is shown in the left panel of Fig. 5. To find the optimum value for $K_{\text {irr }}$, we shifted the spectra by a range of velocities in steps of $0.5 \mathrm{~km} \mathrm{~s}^{-1}$, and computed the integrated intensity for the 22 lines in a $0.1 \AA$ window. The maximum intensity is then found for $222.5 \mathrm{~km} \mathrm{~s}^{-1}$. Fitting a parabola to the central $10 \mathrm{~km} \mathrm{~s}^{-1}$ around the maximum of the peak intensity profile gives the value we adopt for the centre-of-light velocity, $K_{\text {irr }}=222.76 \pm 0.07 \mathrm{~km} \mathrm{~s}^{-1}$.

The second to left panel of Fig. 5 shows the same spectra as in the left panel, after shifting these according to the centreof-light velocity, $K_{\text {irr }}$. Scrutinising this image, we notice some deviations from a linear symmetry. The profile appears to be skewed towards a higher velocity (away from the observer) after the final quadrature point $\varphi=0.75$ and towards a lower velocity (towards the observer) before the first quadrature. This effect can be readily understood using simple geometry. When the hot spot rotates into view after primary eclipse, it first appears on the side that rotates towards us, and so has a negative radial velocity. We only see a complete rotationally broadened line profile when 
M. Vučković et al.: Looking at the bright side of the secondary of AA Dor
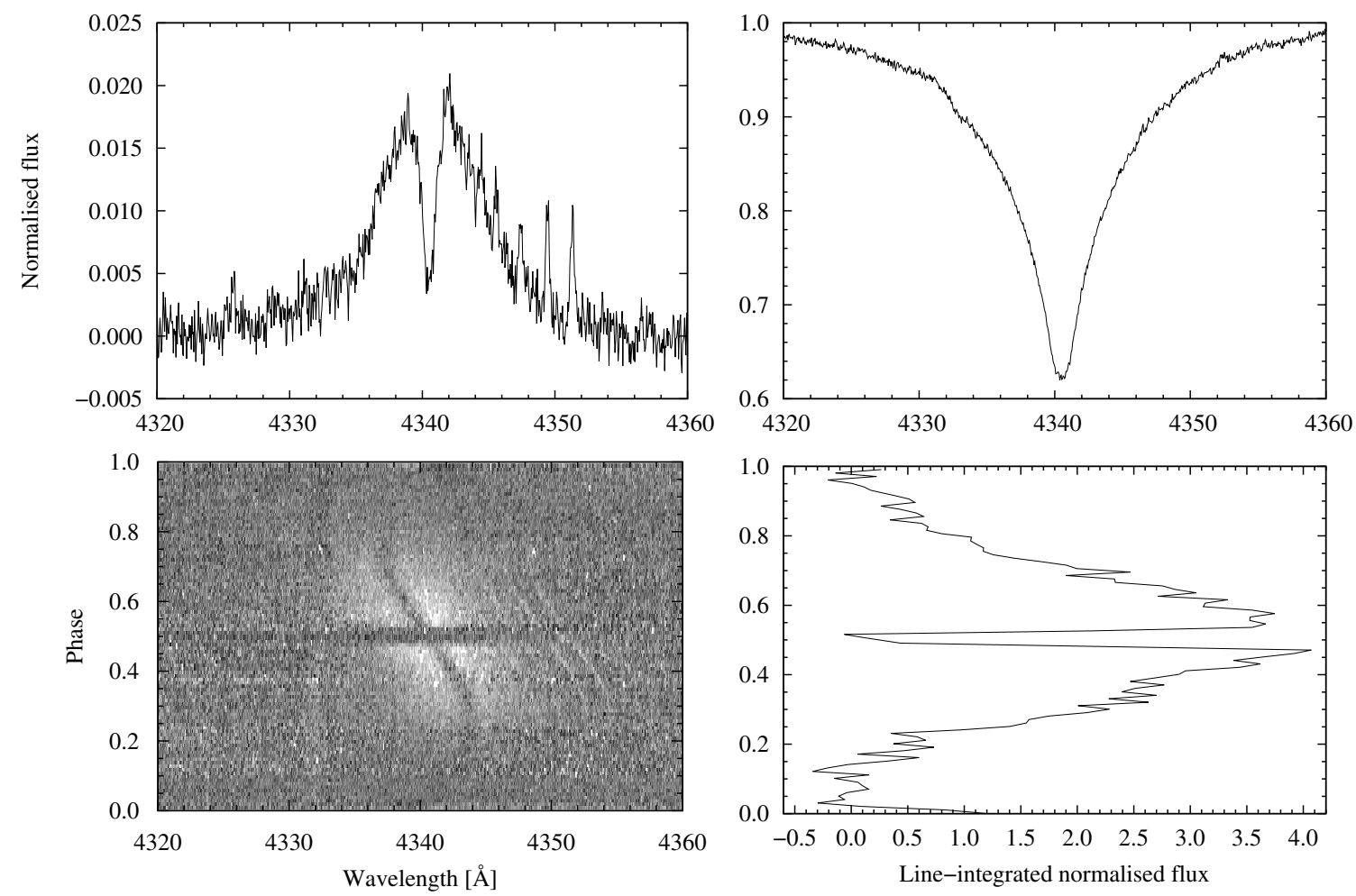

Fig. 2. Greyscale plot (lower-left panel) showing the phase folded spectra in the region around $\mathrm{H} \gamma$, after shifting to the rest frame of the primary and subtracting the mean spectrum (upper-right panel) computed from the least contaminated phases. After shifting to the rest frame of the secondary and summing all phases, the emission profile of the secondary emerges (upper-left panel). Summing each phase-binned spectrum in a window $20 \AA$ wide, and centred on $\mathrm{H} \gamma$ in the rest frame of the secondary, produces the phase profile shown in the lower-right panel.

the spot is completely in view, just before and after secondary eclipse. As the hot spot rotates back onto the far side, the dark side produces a self-eclipse effect that reveals itself as a progressive dimming of the approaching side of the line profile. The last two right hand panels of Fig. 5 show a model line profile based on the stellar radii derived by Hilditch et al. (2003) and our radial velocity measurements, see Table 1 . The third panel shows a model line broadened by a $10 \mathrm{~km} \mathrm{~s}^{-1}$ Gaussian kernel, while the unbroadened one is presented in the fourth panel. It is clear that the model reproduces all the distortion features seen in the observed integrated line profile. The figure also visualises why the measured $K_{\text {irr }}$ must be less than the true centre-of-mass velocity $K_{2}$, since the self-eclipse effect always darkens the line-profile on the side that has the highest velocity in the rest-frame picture.

Another asymmetry feature that emerges in the emission-line profile is the direction of the eclipse of the hotspot in velocity space during secondary eclipse (best seen at the very top of the right panel in Fig. 5). At the beginning of secondary eclipse, at $\varphi=0.48$, we can see that the negative part of the emission profile is obscured, as the primary must cover the side of the secondary that is rotating towards us (negative direction) before it reaches the half that rotates away from the line of sight. At $\varphi=0.52$, the opposite occurs as the side that is rotating towards us emerges before the receding side. This is of course the equivalent of the $\mathrm{RM}$ effect for the hotspot on the irradiated secondary.

\subsection{Determining $K_{\mathrm{dM}}$}

To simulate the spectroscopic features of an irradiated star that are geometrical in origin, we computed some simple numerical models. In addition to the line-profile models shown in the previous section, we computed phase profiles for several different configurations. The models consist of two bodies with the appropriate dimensions, in a circular eclipsing orbit using the parameters for the AA Dor system, as determined by Hilditch et al. (2003). The surface of one body is taken to be dark (the primary), and the other one is dark except for a circular patch with variable radius centred on the axis between the two bodies. In the simplest models we use a straight cut-off radius at a fraction of the radius of the star, while in the "final" model, we tapered the intensity of the light towards the edge of the star so that the brightness of each point was proportional to the area of the irradiating star that is visible above the horizon for that point at a given orbital phase. The phase profiles produced by the simulation were normalised so that they peak at unity just before the start of the eclipse. The resulting phase profiles for a hotspot radius of 50, 75, 90, 95, and 100\% are shown in Fig. 6, together with the "final" model and the integrated Balmer-line phase profile (from the upper-right panel of Fig. 4). Clearly, a hotspot that covers $75 \%$ or less of the irradiated hemisphere disappears behind the rim of the companion around $\varphi=( \pm 0.35)$ much sooner than in the observed profiles. Only profiles of a hotspot covering between 95 and $100 \%$ of the irradiated hemisphere are compatible with the observations. The "final" model seems to fit perfectly at phases close to primary eclipse, but not so well closer to secondary eclipse. However, since there is some arbitrariness regarding the normalisation especially of the peak level at $\varphi=\sim 0.5$, and also the continuum level close to $\varphi=0$, the apparent precision of the fit may be deceptive.

From the irradiated light models, we can also compute the difference between the centre-of-light velocity and the centreof-mass velocity. For a simple hotspot with a radius of 0.975 , we find $K_{\text {irr }}=0.956 K_{2}$. For the "final" model we can use the same 


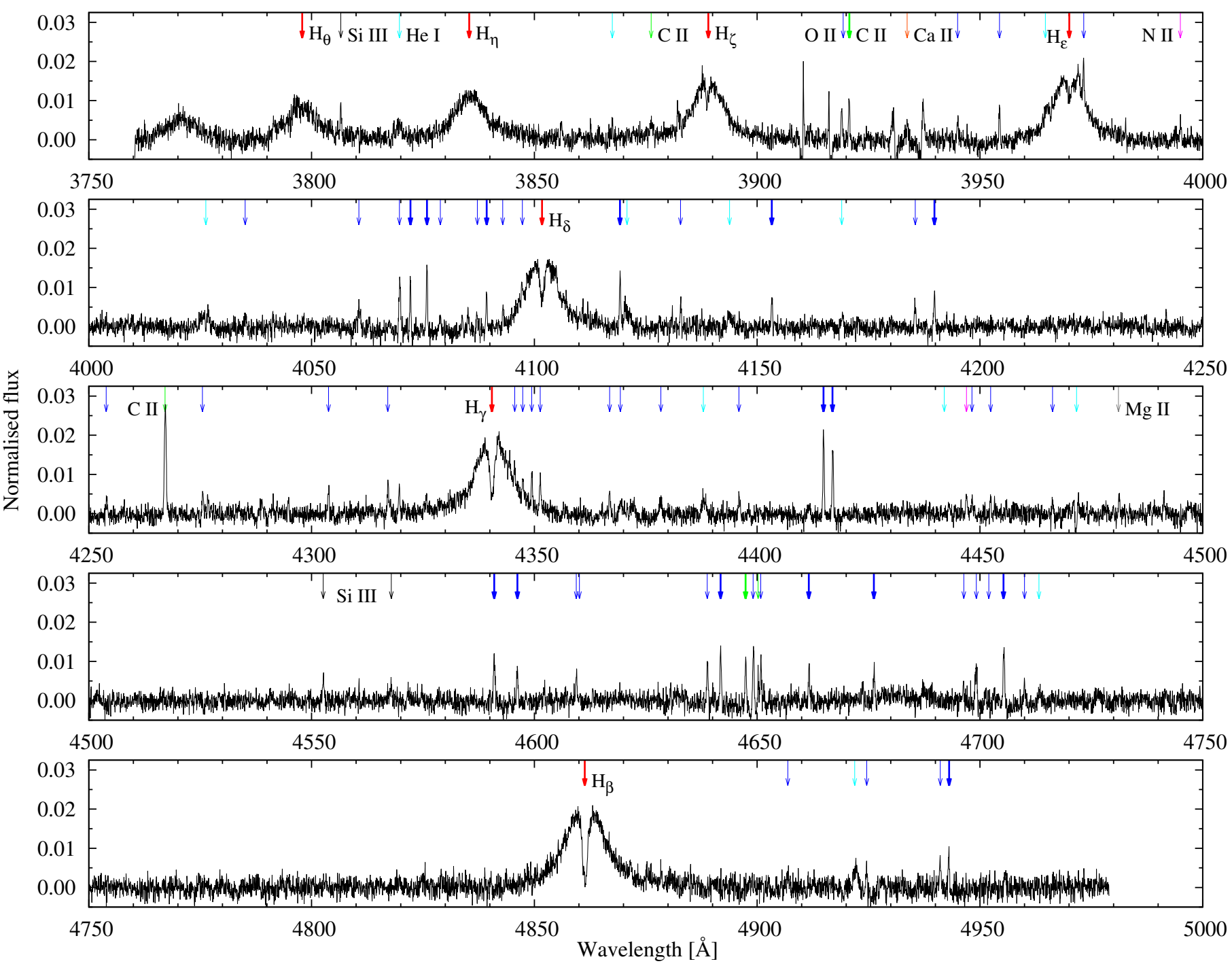

Fig. 3. Emission line spectrum of the secondary. This figure was generated in the same way as for the top-left panel of Fig. 2, but for the full spectroscopic range. The arrows indicate the lines we have identified, while thick arrows indicate those that were used in our radial-velocity analysis. (The color coding on the arrows identifies their species; Balmer lines: red, O II: blue, C II and C III: green, He I: cyan, N II: purple, Si III: black, Mg II: gray.)

procedure that we used to derive $K_{\text {irr }}$ from the observational data. We take the line-profile model shown in the right-hand panel of Fig. 5 and reintroduce a velocity on the order of $K_{\text {irr }}$, after which we locate the velocity shift that produces the maximum intensity of a narrow window across the line. We find that the measured velocity is 0.957 times that introduced to the geometrical model. However, if we use the model profile subjected to Gaussian broadening, we get a slightly smaller reduction factor of 0.963 . We take the latter value as the most likely value for the correction factor, $K_{\text {irr }} / K_{2}$. The error on this number is not easy to quantify. From the differences between a sharp and broadened model we estimate it to be on the order of $0.3 \%$ and conclude that the centre-of-mass velocity for the secondary is

$K_{2}=\frac{K_{\text {irr }}}{0.963 \pm 0.003}=231.3 \pm 0.7 \mathrm{~km} \mathrm{~s}^{-1}$

This results in a mass ratio

$q=\frac{K_{2}}{K_{\mathrm{sdO}}}=0.171 \pm 0.001$

A146, page 6 of 12
The masses for the two components then follow,

$$
\begin{aligned}
M_{\mathrm{sdO}} & =1.036 \times 10^{-7}\left(\frac{K_{2}}{\sin i}\right)^{3}(q+1)^{2} P=0.46 \pm 0.01 M_{\odot} \\
M_{2} & =q M_{\mathrm{sdO}}=0.079 \pm 0.002 M_{\odot}
\end{aligned}
$$

implying a canonical mass for the hot subdwarf and a mass just at the substellar limit for the companion (Baraffe et al. 2015).

\section{Modelling the spectrum of the irradiated secondary}

Irradiation effects are strong in close, HW-Vir-like subdwarf binaries with cool companions. In non-eclipsing binaries the reflection effect usually completely dominates the light curve. In the AA Dor system, the primary is a hot sdO subdwarf, and hence the companion is subjected to a more extreme UV irradiation. Light curve modelling codes like the Wilson-Devinney variants (e.g. Wilson \& Devinney 1971, or PHOEBE Degroote et al. 2013) assume that the flux variation is due to reflection off 
M. Vučković et al.: Looking at the bright side of the secondary of AA Dor
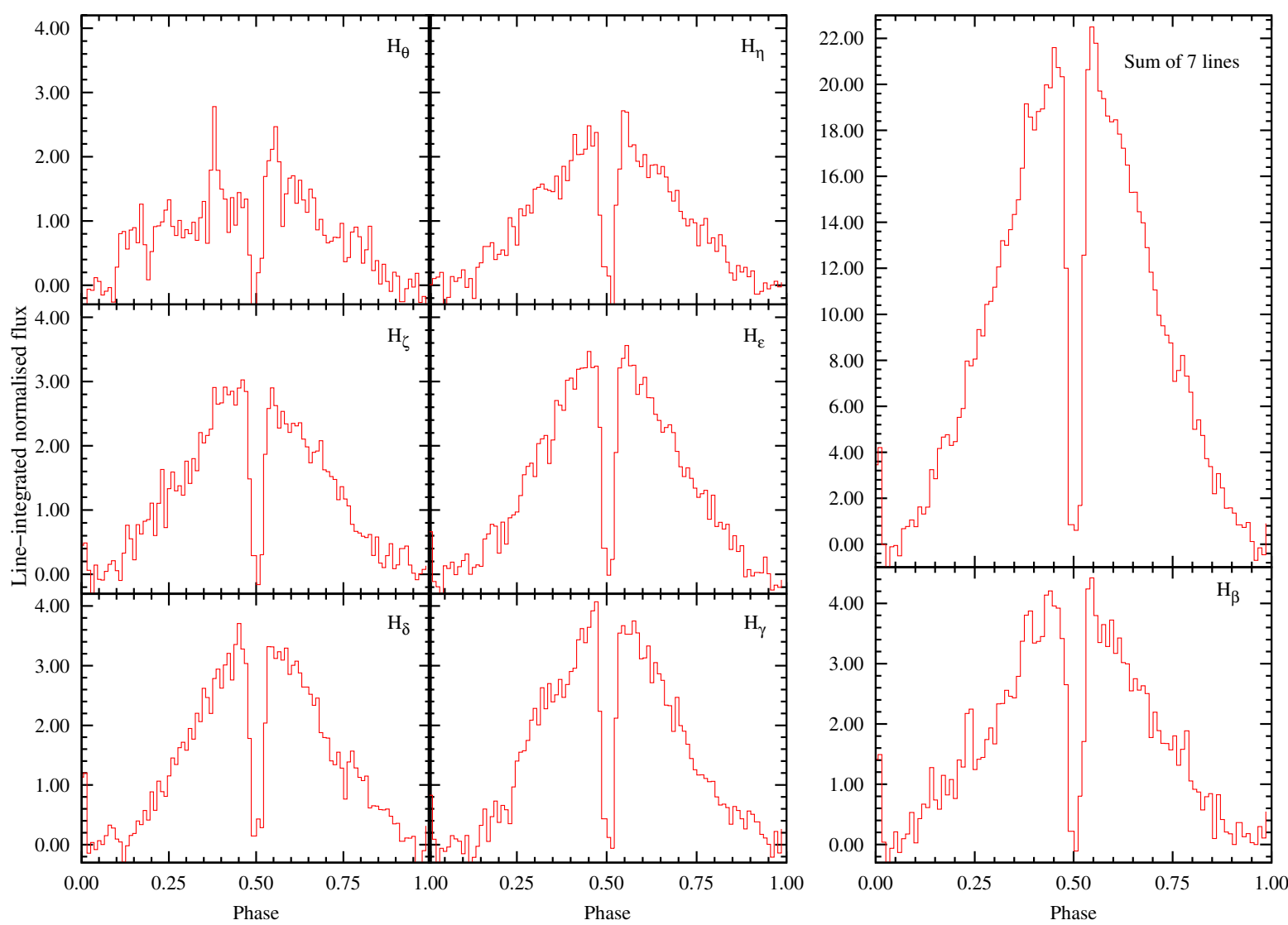

Fig. 4. Phase profiles for all the Balmer lines produced in the same way as for $\mathrm{H} \gamma$ in the lower-right panel of Fig. 2, and the sum of all 7 Balmer lines (upper-right panel).

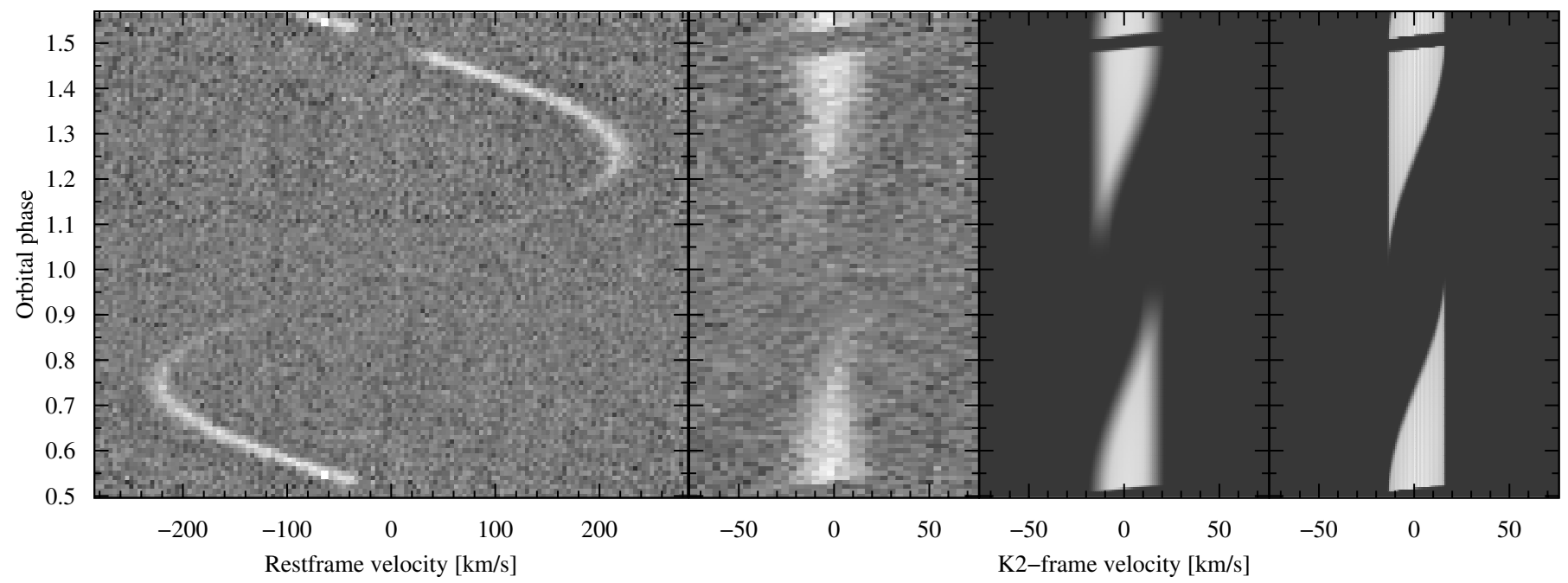

Fig. 5. Greyscale plot of the sum of all the metal lines in the rest frame of the system (left) and shifted to the rest frame of the secondary (middle). The last two panels show a geometrical model-line profile for comparison, with the first having been subjected to a Gaussian broadening.

the surface of the secondary or they include some form of energy redistribution on the companion. While this approach can be conveniently parametrised with only a few parameters and gives a very good quantitative description of the light curve, it does not provide the physical conditions in the atmosphere of the companion. To fully understand the evolution of close binaries, we must investigate the reflection effect. By understanding the radiative interactions between the members of close binaries, we can derive accurate surface parameters and better interpret their high precision photometric data, such as the Kepler eclipsing binary light curves (Prša et al. 2011). This methodology will eventually lead to the characterisation of non-transiting exoplanets based on spectral models and measured reflection light curves.

To model the effects of irradiation and reproduce both the spectral variations and the reflection effect in the light curve of AADor, we followed the description of the geometry of eclipsing binaries developed by Günther \& Wawrzyn (2011) and Wawrzyn et al. (2009). This model provides the visible fractions 


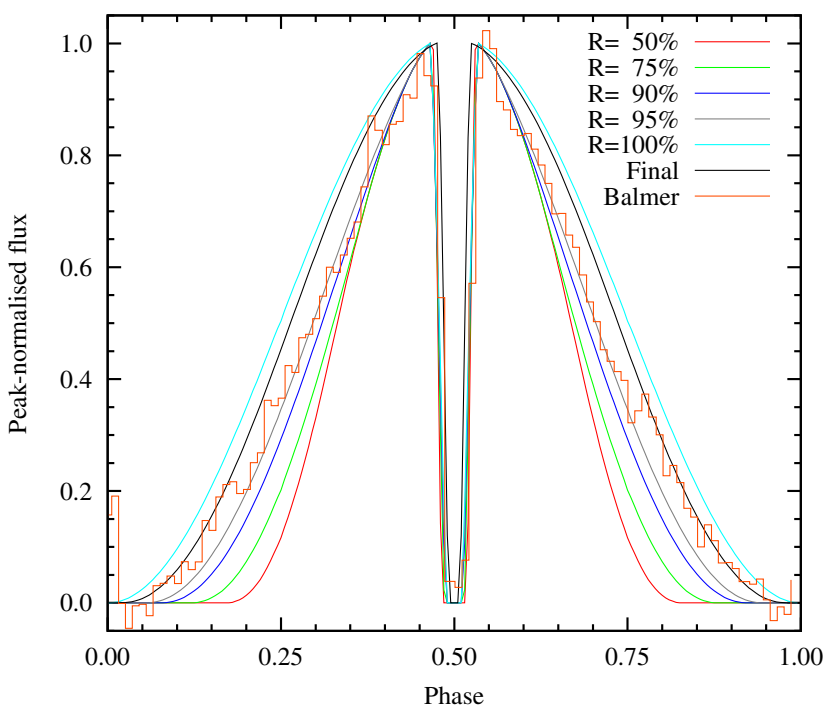

Fig. 6. Normalised phase profiles from models of emission from hotspots of various radii (curves). The 'final' model includes edge effects and limb darkening. Also shown, are the observed phase profiles from the sum of Balmer and metal lines. It is clear from comparing the model and observed profiles that the size of the hotspot must be between 95 and $100 \%$.

of equally irradiated zones on the companion as a function of orbital phase. For compatibility with Günther \& Wawrzyn (2011), we adopted the same spherical coordinate system with the origin at the centre of the secondary star and the $z$-axis intersecting the centre of the primary. The $z$-axis intersects the surface of the companion at the substellar point, where irradiation is the strongest. Constant latitudes $\theta$ on the secondary (measured from the $z$-axis) receive the same amount of incident flux in this model. For simplicity, we calculated our spectral model for the orbital phase of maximum intensity $(\varphi=0.23-0.77)$ to match the phase coverage of the extracted spectrum. We used three zones that cover the area between $\theta=0-20^{\circ}, 20-50^{\circ}$, and $50-90^{\circ}$ on the surface of the companion. The size of the irradiated surface is determined by the relative radii of the stars and the semi-major axis of the system. The twilight zone (transition region between the day and night sides) is less irradiated by the primary, so here we scaled the dilution factor by the relative visible area of the disk of the primary. The night side does not contribute to the spectrum in this model. Next, we calculated plane-parallel irradiated non-LTE model atmospheres with TLUSTY (Hubeny \& Lanz 1995) and synthetic spectra with SYNSPEC (Lanz \& Hubeny 2007), and reproduced the spectrum of the companion with a superposition of the irradiated concentric zones. The synthetic spectra of each zone were co-added with relative weights: $0.08,0.37$, and 0.55 . This corresponds to the inclination of $89.21^{\circ}$ and considers the phaseintegrated contributions of the zones between $\varphi=0.23-0.77$. All our models were calculated with opacity sampling with $\mathrm{H}$, $\mathrm{He}$, and CNO composition and model atoms taken from the TLUSTY web page ${ }^{3}$. We assumed that the irradiating flux is black body-like, with $T_{\text {eff }}=42000 \mathrm{~K}$ (Klepp \& Rauch 2011). The dilution factor describes the irradiation efficiency, that is, the fraction of the irradiating flux absorbed by the companion. The dilution factor differs in our model from the geometric dilution factor (Mihalas 1978) as it depends on several other parameters as well. It is affected by the system geometry, the irradiation angle, as well as the spectral properties of the irradiating source. In

\footnotetext{
3 http://nova.astro.umd.edu/
}

our analysis, therefore, the dilution factor is a free parameter that includes other poorly constrained or degenerate parameters. The effective temperature of the irradiated layers in similar systems are reported to be in the range of $13000-15000 \mathrm{~K}$ (e.g. HW Vir, $T_{\mathrm{sdB}}=28000 \mathrm{~K}$, Kiss et al. 2000), which our models reproduce with a dilution factor of 0.021 . From the relative radii and distance of the stars, the geometric dilution factor is 0.006 at the substellar point. However, probably because of departures of the UV sdO spectrum from a black-body spectrum, the best model fit required a higher dilution factor of 0.06 in the $\theta=0-20^{\circ}$ zone. In the subsequent zones we used 0.049 and 0.021 , which were scaled with $\cos \theta$. Further details of the geometry can be found in Günther \& Wawrzyn (2011).

The model presented here is our first attempt to reproduce the spectrum of the companion, therefore several simplifying approximations have been made. The spectral model does not include a magnetic field, spots and clouds, or molecular opacities. We also neglected convection. While low-mass stars are fully convective, this approximation is valid for the bright side where irradiation supresses convection (Podsiadlowski 1991). Both stars are assumed to be spherical in a circular orbit. Our model does not include any horizontal energy transport that would distribute energy from the substellar point towards the night side. Although such a meridional flow is plausible at strong irradiation and would explain the homogeneity of the irradiated hemisphere (Beer \& Podsiadlowski 2002), the current UVES observations are not suitable for such a study.

We fit the observed spectra in Fig. 3 with our iterative steepest-descent spectral-fitting procedure XTGRID (Németh et al. 2012). The structure of the irradiated atmosphere of the secondary star in AA Dor resembles an inverted B-type star, and therefore it can be modelled with the same techniques as hot stars. Based on the observed radius $R=0.11 R_{\odot}$, if the companion is a red dwarf, its night side temperature is $\sim 2400 \mathrm{~K}$; if it is below the hydrogen burning limit the temperature may be as low as $\sim 1700 \mathrm{~K}$ (Dieterich et al. 2014). Therefore we fixed the effective temperature of the secondary at $T=2000 \mathrm{~K}$ and the surface gravity had a starting value of $\log g=5.25 \mathrm{~cm} \mathrm{~s}^{-2}$, which is consistent with the photometric value (Hilditch et al. 2003). The gravity, abundances, and the dilution factor were adjusted iteratively for the substellar point and the dilution factor was scaled geometrically in the subsequent zones. We assumed a homogeneous abundance distribution in the atmosphere and adjusted the abundances globally for the entire irradiated hemisphere. We calculated three model atmospheres to the different irradiation zones and their corresponding synthetic spectra in every iteration. The zonal spectra have been normalised, weighted, and co-added, and the final spectrum shifted to the data as shown in Fig. 7. Our fit was based on the Balmer and metal emission lines, which explains the virtually negative flux in the figure. Data points, such as the Balmer line self-absorption cores and far line wings, where the synthetic spectrum reaches negative values (below the continuum level) were taken out of the chisquare calculation by the fit procedure. We applied a $20 \mathrm{~km} \mathrm{~s}^{-1}$ rotational broadening to approximate the smearing caused by the assumed tidally-locked rotation of the companion and extraction of the spectrum in velocity space. The rotational broadening profile is symmetric only near the secondary eclipse. We found that, while a single irradiation zone with different dilution factors and system geometries can reproduce parts of the spectrum, only a multi-zone model could address the majority of the features simultaneously. We conclude, in agreement with Günther \& Wawrzyn (2011), that a single temperature model cannot reproduce the spectrum of the irradiated hemisphere in detail, even 
M. Vučković et al.: Looking at the bright side of the secondary of AA Dor

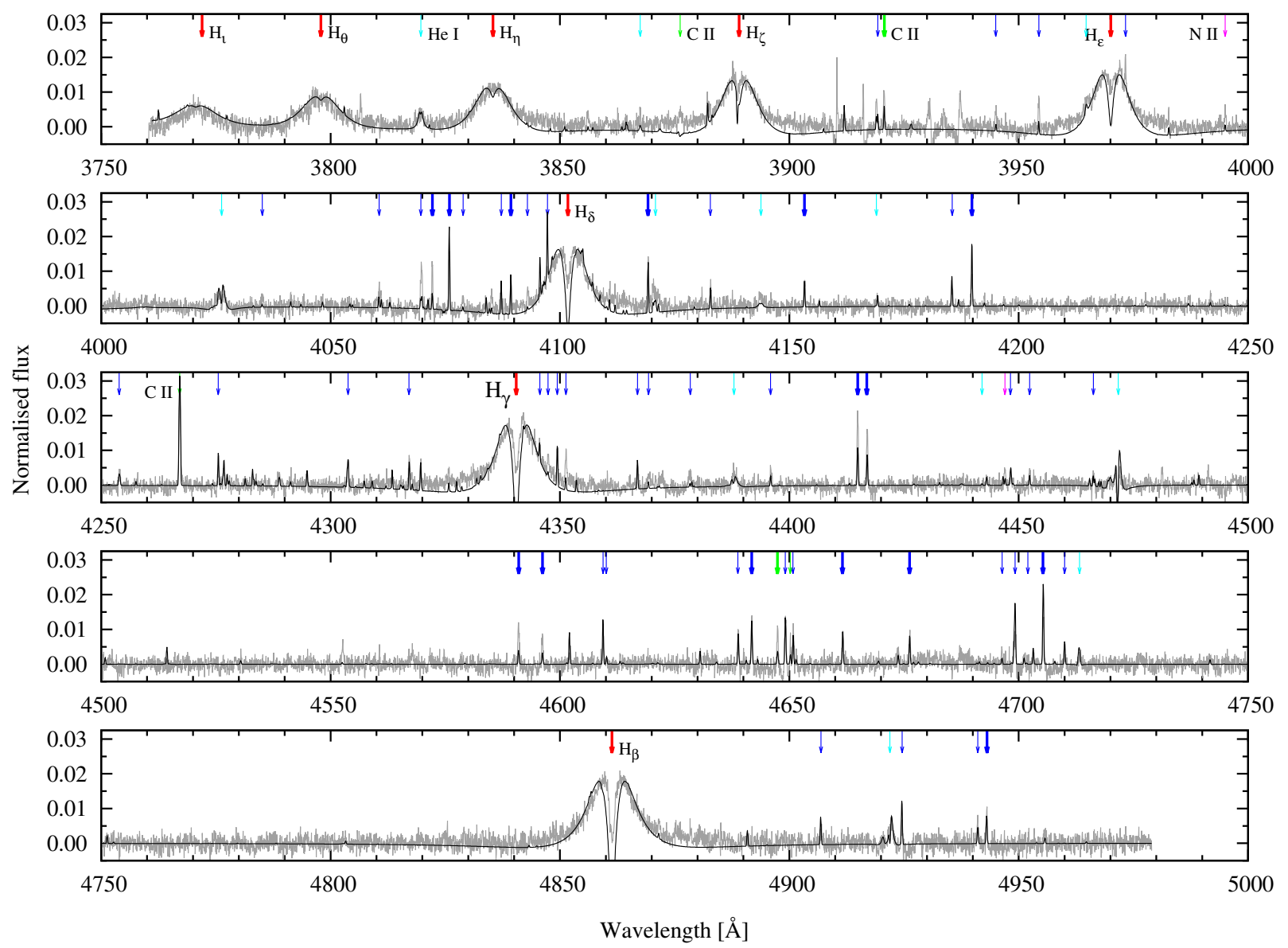

Fig. 7. Synthetic emission line spectrum of the dM secondary. Lines coincide with the ones shown in Fig. 3. (The colour coding for the arrows identifies their species; Balmer lines: red, O II: blue, C II and C III: green, He I: cyan, N II: purple)

though Fig. 6 suggests that the hotspot is largely uniform and covers the entire inner hemisphere of the secondary. The models are very insensitive to the effective temperature of the companion, but the Balmer lines are sensitive to the surface gravity and the dilution factor. This result is not surprising because the atmospheric temperature structure is dominated by the irradiation that penetrates below the observed photosphere. The Balmer profiles are broad and shallow in the substellar point and become narrower and stronger toward the terminator. Therefore the line profiles also depend on the geometry and surface integration, which makes determining a precise surface gravity complicated. We found surface gravities in the range of $\log g=5.05-5.45 \mathrm{~cm} \mathrm{~s}^{-2}$ in the three zones. The surface gravity of the companion can be precisely estimated in eclipsing binaries from the orbital parameters (Southworth et al. 2004). Using the results of Hilditch et al. (2003) and the radial velocity amplitude derived in Sect. 4, we found $\log g=5.276 \pm 0.002 \mathrm{~cm} \mathrm{~s}^{-2}$, although this method does not take irradiation effects into account. The observed weak absorption in the wings of Balmer lines indicate that the surface gravity may depend on the distance from the substellar point, which needs further investigation.

The parameters of our best-fit model are listed in Table 2 . Based on the measured CNO abundances, we estimate the metallicity of the secondary to be $[M / H]=-0.7$ dex. This metallicity is surprisingly high and most likely does not reflect the primordial metallicity of the companion. The C- and O-rich, $\mathrm{N}$-depleted abundance pattern resembles the sdO primary (Fleig et al. 2008). Accretion from a radiatively-driven optically-thin wind of the sdO (Krtička \& Kubát 2010) is a plausible reason for the observed high metallicity and needs further investigation.

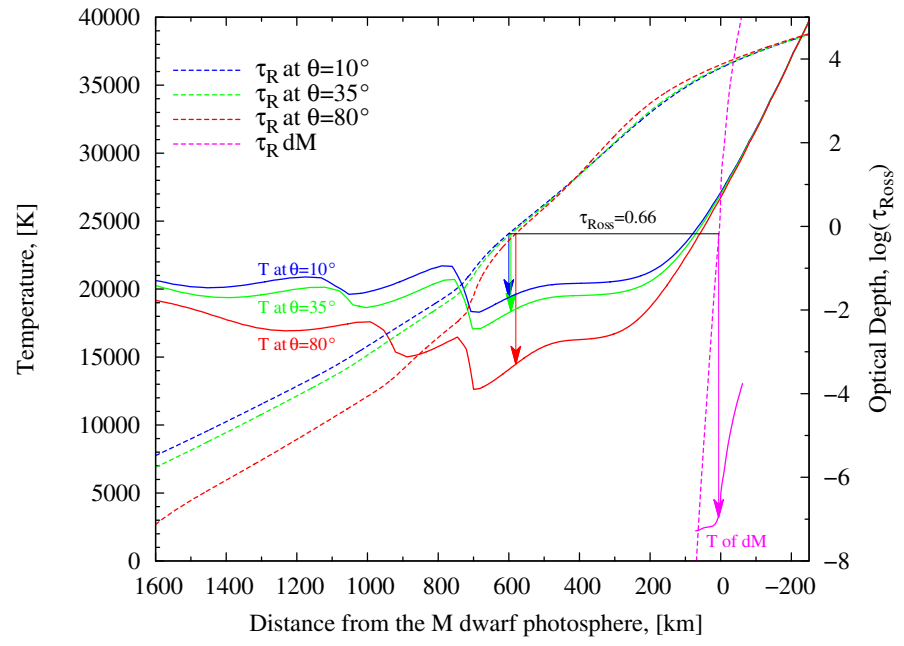

Fig. 8. Temperature structure of the atmosphere of the irradiated dM secondary star in the AADor system. The three irradiated zones for $\theta=10^{\circ}, 35^{\circ}$, and $80^{\circ}$ are shown together with the structure of the unperturbed $\mathrm{dM}$ on the night side. The irradiated zones show several temperature inversion regions and an extension of the atmosphere. The photospheres are progressively higher and hotter toward the substellar point with respect to the dark side, as indicated by the arrows at $\tau_{\text {Ross }}=0.66$.

The consistent metal and Balmer line radial velocities exclude a circumstellar origin of the metal lines.

TLUSTY provides the atmospheric temperature, density, and pressure structure that allowed us to estimate the extension of the atmosphere. Figure 8 shows the vertical temperature structure 

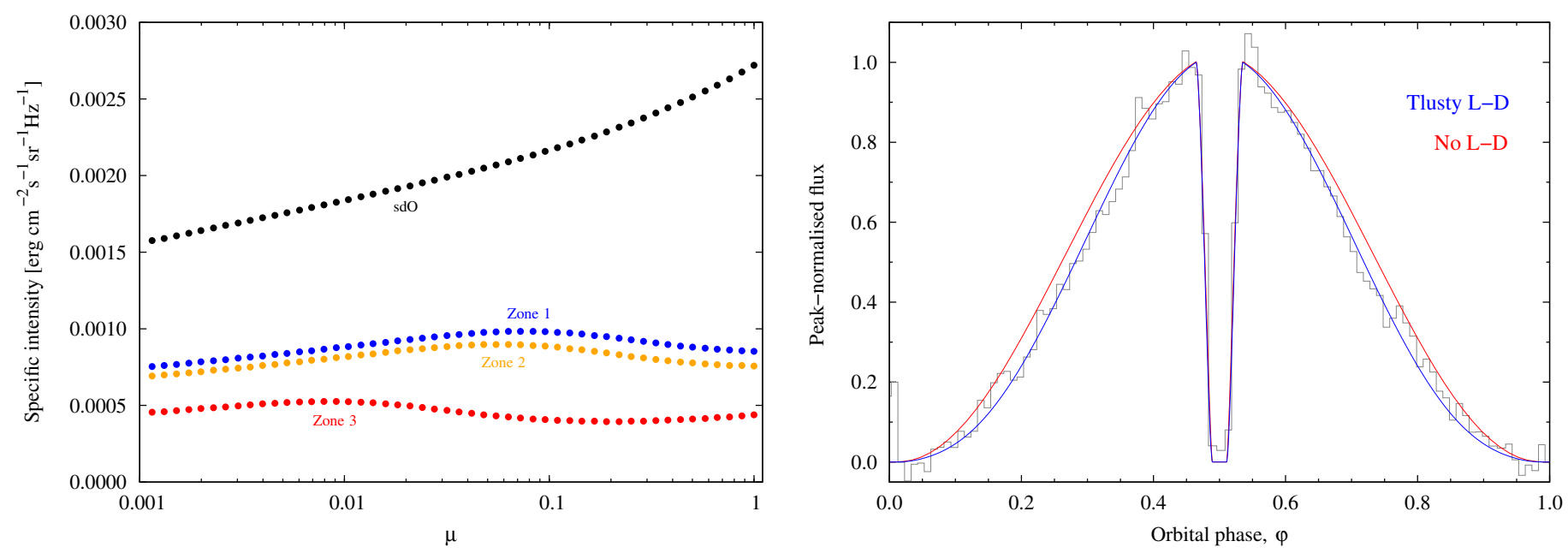

Fig. 9. Left: specific intensities for the sdO star and for the three irradiated zones on the M dwarf. Note, we use a logarithmic scale on the abscissa to better represent the centre-to-edge variations of the specific intensities, $\mu$ is the cosine of the polar angle (between the line of sight and the surface normal at a given point on the companion) that takes the value 1 in the disk centre and 0 on the edge. There is only a little difference between the substellar zone (No. 1) and the intermediate zone (No. 2), but the terminator zone (No. 3) is substantially fainter. Each zone shows a moderate brightening towards the limb. Right: effects of limb darkening on the phase profile. The three-zone TLUSTY model only provides a marginally better fit than a flat surface intensity distribution of the companion.

Table 2. Atmospheric parameters of the irradiated $M$ dwarf in AA Dor.

\begin{tabular}{|c|c|c|c|c|}
\hline \multicolumn{2}{|l|}{ Parameter } & $\begin{array}{l}\text { Zone } 1 \\
\theta<20^{\circ}\end{array}$ & $\begin{array}{c}\text { Zone } 2 \\
20^{\circ}<\theta<70^{\circ}\end{array}$ & $\begin{array}{l}\text { Zone } 3 \\
70^{\circ}<\theta\end{array}$ \\
\hline \multicolumn{2}{|c|}{$T_{\text {eff }}(\mathrm{K})$ day side } & 19200 & 18300 & 14300 \\
\hline \multicolumn{2}{|c|}{ Dilution factor } & 0.060 & 0.049 & 0.021 \\
\hline \multirow{2}{*}{\multicolumn{2}{|c|}{$\begin{array}{l}T_{\text {eff }}(\mathrm{K}) \text { night side } \\
\log g\left(\mathrm{~cm} \mathrm{~s}^{-2}\right)\end{array}$}} & & $2000 \pm 500$ & \\
\hline & & & $5.25 \pm 0.25$ & \\
\hline \multicolumn{2}{|c|}{$F_{\mathrm{dM}} / F_{\mathrm{sdO}}($ at $5000 \AA, \varphi=0.47)$} & & $0.07 \pm 0.02$ & \\
\hline Abundances & $\log (n \mathrm{X} / n \mathrm{H})$ & + & - & $\times$ Solar \\
\hline $\mathrm{He}$ & -1.69 & 0.88 & 0.38 & 0.24 \\
\hline $\mathrm{C}$ & -4.30 & 0.02 & 0.11 & 0.19 \\
\hline $\mathrm{N}$ & -4.89 & 0.06 & 0.04 & 0.19 \\
\hline $\mathrm{O}$ & -3.94 & 0.01 & 0.03 & 0.23 \\
\hline
\end{tabular}

Notes. Surface abundances are also listed with respect to the solar abundances (Asplund et al. 2009).

of the three irradiated zones. We found inversion layers where the temperature changes rapidly with optical depth. By stacking plane-parallel models with XTGRID, we could account for sphericity effects. The optical depth scale was integrated inwards and synthetic spectra were calculated at subsequently higher layers towards the limb. The differences among the zones in photospheric depths are relatively small. We found that the pressurebroadened Balmer emission-line wings form deeper than the line core and most of the metal lines. The steep temperature gradients in the inversion layers and line forming region provide a spectral diversity to the zones. With CoOLTLUSTY, an optimised version of the code for brown dwarf and planetary atmospheres (Hubeny et al. 2003), we calculated a dM model for the night side of the companion. The pressure scale height was found to be $8 \mathrm{~km}$ for the night side and $130 \mathrm{~km}$ in the substellar zone, suggesting an extended atmosphere. Interestingly, these scale heights are comparable to those of the Earth and the Sun. By matching the photospheric pressure of the M dwarf on the dark side with the pressure in the irradiated zones, we shifted the zones to the same base. This allowed us to measure the thickness of the atmosphere in the zones. We found that the irradiated zones have a larger radius by about $600 \mathrm{~km}$, which is about $0.8 \%$ of the radius of the secondary. Similar expansions have been observed in irradiated extrasolar giant planets (Burrows et al. 2007).

From the spectral models, the limb darkening of the irradiated hemisphere can be evaluated numerically. The left panel of Fig. 9 shows the limb darkening of the three irradiated zones and of the sdO star for comparison. The actual surface flux distribution is not only function of the angle to the surface normal, but also depends on the orbital phase and distance from the substellar point. Therefore, we developed a model that allows us to use the specific intensities and surface integration in the phaseprofile calculation. With this model the total surface intensity of the visible part of the irradiated hemisphere can be calculated for an arbitrary orbital phase and the light curve of the secondary can be obtained. Again, we used the published photometric radii, inclination, and semi-major axis of the system (Hilditch et al. 2003). The right panel of Fig. 9 shows that the model reproduced the observed phase curve quite well. The model also reproduced the size of the spot, which can be inferred from the phase coverage of the reflection effect, in good agreement with the analysis in Sect. 4.2. The phase curve shows some extra flux between $\varphi=0.75-0.95$. We interpret this excess flux as the signature of an asymmetry in the hotspot, resulting from a non-synchronous rotation of the companion with the orbit. The Roche potential of the companion does not suggest a notable deformation, but a small atmospheric expansion near the substellar point is likely due to irradiation. However, its effect on the phase curve must be negligible since an ellipsoidal-like variation would give a maximal contribution at $\varphi=0.25$, which is not obvious in Fig. 9 .

To overcome the limitations of our current models, we will focus on three major improvements in the future: (1) replacing the black-body irradiation with the spectrum of the sdO star; (2) the implementation of a more adjustable surface intensity distribution that will allow us to investigate the asymmetries of the hotspot and (3) improving the consistency between the spectral and light-curve models with the help of optical-infrared photometry. A better consistency may also require more irradiation zones and opacity sources. This new model will allow us to interpret high precision light curves and infer the conditions in the atmospheres of irradiated companions in faint reflection effect 
binaries, where direct spectroscopy is not yet feasible. We will present our progress in a forthcoming publication.

\section{Discussion and summary}

We have reanalysed the UVES dataset of the AA Dor binary system, with particular attention to extracting orbital phase information from the emission lines of the irradiated secondary. We integrated the signal from the Balmer lines and 22 narrow emission lines and were able to firmly establish that the emission region covers no less than $95 \%$ of the irradiated hemisphere. With this information we were able to accurately translate the observed centre-of-light velocity, $K_{\text {irr }}$, into a precise centre-of-mass velocity, $K_{2}$. With this last piece of the puzzle in place, we were able to finally establish the masses of the two components with very high precision. The new numbers are fully compatible with the results of Hoyer et al. (2015), but with error bars that are five to ten times lower.

With radial velocities of the primary, $K_{\text {sdO }}=-39.63 \pm$ $0.21 \mathrm{~km} \mathrm{~s}^{-1}$ and the secondary, $K_{2}=231.3 \pm 0.7 \mathrm{~km} \mathrm{~s}^{-1}$, we get the mass for the primary, $M_{\mathrm{sdO}}=0.46 \pm 0.01 M_{\odot}$, and the secondary, $M_{2}=0.079 \pm 0.002 M_{\odot}$. These masses are in full agreement with Klepp \& Rauch (2011) and Hoyer et al. (2015), which confirms the hot subdwarf primary star and tightly confines the secondary to the hydrogen burning mass limit, i.e. either a brown dwarf or a late dM star (Baraffe et al. 2015).

To further study the nature of the secondary star in AA Dor, we need to obtain high $\mathrm{S} / \mathrm{N}$ near-infrared (NIR) spectra from both the cold and hot hemisphere. The recent attempt to obtain such data by Hoyer et al. (2015) was not successful since poor observing conditions prevented them from detecting any signal from the secondary in the NIR part of the X-Shooter timeresolved data set. We strongly encourage repeating this attempt in excellent observing conditions.

Combining the 22 metal lines of the secondary to study the shape of the emission velocity profiles we detected two distinct asymmetry features. The velocity profiles appear skewed at the quadratures which, because of the pure geometrical effect, produces a sort of a self-eclipse. The asymmetry in the velocity profiles that emerges at the secondary eclipse is caused by the rotation of the secondary, and hence we termed it as the RM effect of the hotspot.

Furthermore, we have modelled the atmosphere of the secondary of AA Dor, including the irradiation effects that matched the observed spectrum of the companion very well. Comparing the synthetic and observed spectra we confirmed the majority of the emission lines as listed in Table A.1. While the hot spot covers almost the entire inner hemisphere, a single temperature model fails to reproduce the observed irradiated spectrum, which indicates that, while there must be a very effective process distributing the energy over the irradiated hemisphere, the irradiated regions contribute differently to the spectrum of the companion. The irradiated zones show several temperature inversion regions and an extension of the atmosphere.

We developed a model that allows us to use a surface integration of the specific intensities in the phase-profile calculation. With this model the total surface intensity of the visible part of the hotspot can be calculated for an arbitrary orbital phase and the light curve of the secondary can be obtained.

The observations of irradiated planets are indicative of the heat transport from the day side to the night side (Knutson et al. 2007). However, to understand the circulation patterns of hot Jupiters, it is crucial to analyse the temperature inversion in the upper atmospheres. While we are witnessing the era of hot Jupiters and circumbinary planets around evolved binaries, AA Dor stands as a bona fide system to understand the radiation transport in the extreme conditions of irradiated (sub)stellar objects.

Acknowledgements. We thank Cedric Ledoux for helpful discussions on UVES data reduction. We thank Tom Marsh for the use of his spectrum analysis program Molly. This research was based on observations collected at the European Southern Observatory, (Chile), Program ID: 066.D-1800. This research made use of SIMBAD database, operated at the CDS (Strasbourg, France) and NASA Astrophysics Data System Bibliographic Services.

\section{References}

Asplund, M., Grevesse, N., Sauval, A. J., \& Scott, P. 2009, ARA\&A, 47, 481 Baraffe, I., Homeier, D., Allard, F., \& Chabrier, G. 2015, A\&A, 577, A42 Barlow, B. N., Kilkenny, D., Drechsel, H., et al. 2013, MNRAS, 430, 22 Barman, T. S., Hauschildt, P. H., \& Allard, F. 2004, ApJ, 614, 338 Bear, E., \& Soker, N. 2011, MNRAS, 414, 1788

Beer, M. E., \& Podsiadlowski, P. 2002, in Exotic Stars as Challenges to Evolution, eds. C. A. Tout, \& W. van Hamme, ASP Conf. Ser., 279, 253 Beuermann, K., Hessman, F. V., Dreizler, S., et al. 2010, A\&A, 521, L60 Beuermann, K., Breitenstein, P., Debski, B., et al. 2012a, A\&A, 540, A8 Beuermann, K., Dreizler, S., Hessman, F. V., \& Deller, J. 2012b, A\&A, 543, A138

Burrows, A., Hubeny, I., Budaj, J., \& Hubbard, W. B. 2007, ApJ, 661, 502

Charpinet, S., Fontaine, G., Brassard, P., et al. 2011, Nature, 480, 496

Copperwheat, C. M., Morales-Rueda, L., Marsh, T. R., Maxted, P. F. L., \& Heber, U. 2011, MNRAS, 415, 1381

Degroote, P., Conroy, K., Hambleton, K., et al. 2013, in EAS Publ. Ser., 64, 277

Dieterich, S. B., Henry, T. J., Jao, W.-C., et al. 2014, AJ, 147, 94

Dorman, B., Rood, R. T., \& O'Connell, R. W. 1993, ApJ, 419, 596

Driebe, T., Schoenberner, D., Bloecker, T., \& Herwig, F. 1998, A\&A, 339, 123

Fleig, J., Rauch, T., Werner, K., \& Kruk, J. W. 2008, A\&A, 492, 565

Geier, S., Marsh, T. R., Wang, B., et al. 2013, A\&A, 554, A54

Günther, H. M., \& Wawrzyn, A. C. 2011, A\&A, 526, A117

Han, Z., Podsiadlowski, P., Maxted, P. F. L., Marsh, T. R., \& Ivanova, N. 2002, MNRAS, 336, 449

Han, Z., Podsiadlowski, P., Maxted, P. F. L., \& Marsh, T. R. 2003, MNRAS, 341, 669

Heber, U. 2009, ARA\&A, 47, 211

Heber, U., Hunger, K., Jonas, G., \& Kudritzki, R. P. 1984, A\&A, 130, 119

Hilditch, R. W., \& Kilkenny, D. 1980, MNRAS, 192, 15P

Hilditch, R. W., Harries, T. J., \& Hill, G. 1996, MNRAS, 279, 1380

Hilditch, R. W., Kilkenny, D., Lynas-Gray, A. E., \& Hill, G. 2003, MNRAS, 344,644

Hoyer, D., Rauch, T., Werner, K., Hauschildt, P. H., \& Kruk, J. W. 2015, A\&A, 578, A125

Hubeny, I., \& Lanz, T. 1995, ApJ, 439, 875

Hubeny, I., Burrows, A., \& Sudarsky, D. 2003, ApJ, 594, 1011

Iben, Jr., I., \& Tutukov, A. V. 1984, ApJS, 54, 335

Kawka, A., Vennes, S., O’Toole, S., et al. 2015, MNRAS, 450, 3514

Kilkenny, D. 2014, MNRAS, 445, 4247

Kilkenny, D., \& Hill, P. W. 1975, MNRAS, 173, 625

Kilkenny, D., Hill, P. W., \& Penfold, J. E. 1981, MNRAS, 194, 429

Kiss, L. L., Csák, B., Szatmáry, K., Furész, G., \& Sziládi, K. 2000, A\&A, 364, 199

Klepp, S., \& Rauch, T. 2011, A\&A, 531, L7

Knutson, H. A., Charbonneau, D., Allen, L. E., et al. 2007, Nature, 447, 183

Krtička, J., \& Kubát, J. 2010, Ap\&SS, 329, 145

Kudritzki, R. P., Simon, K. P., Lynas-Gray, A. E., Kilkenny, D., \& Hill, P. W. 1982, A\&A, 106, 254

Lanz, T., \& Hubeny, I. 2007, ApJS, 169, 83

Lohr, M. E., Norton, A. J., Anderson, D. R., et al. 2014, A\&A, 566, A128

Maxted, P. f. L., Heber, U., Marsh, T. R., \& North, R. C. 2001, MNRAS, 326, 1391

Mihalas, D. 1978, Stellar atmospheres, 2nd edn.

Morales-Rueda, L., Maxted, P. F. L., Marsh, T. R., North, R. C., \& Heber, U. 2003, MNRAS, 338, 752

Morales-Rueda, L., Maxted, P. F. L., Marsh, T. R., Kilkenny, D., \& O’Donoghue, D. 2006, Balt. Astron., 15, 187

Napiwotzki, R., Karl, C. A., Lisker, T., et al. 2004, Ap\&SS, 291, 321

Németh, P., Kawka, A., \& Vennes, S. 2012, MNRAS, 427, 2180

Paczynski, B., \& Dearborn, D. S. 1980, MNRAS, 190, 395 
Pápics, P. I., Briquet, M., Baglin, A., et al. 2012, A\&A, 542, A55

Podsiadlowski, P. 1991, Nature, 350, 136

Prša, A., Batalha, N., Slawson, R. W., et al. 2011, AJ, 141, 83

Rauch, T., \& Werner, K. 2003, A\&A, 400, 271

Rossiter, R. A. 1924, ApJ, 60, 15

Saffer, R. A., Bergeron, P., Koester, D., \& Liebert, J. 1994, ApJ, 432, 35

Schaffenroth, V., Geier, S., Drechsel, H., et al. 2013, A\&A, 553, A18

Schaffenroth, V., Geier, S., Heber, U., et al. 2014, A\&A, 564, A98

Schaffenroth, V., Barlow, B. N., Drechsel, H., \& Dunlap, B. H. 2015, A\&A, 576, A123

Schenker, K. 2005, in 14th European Workshop on White Dwarfs, eds. D. Koester, \& S. Moehler, ASP Conf. Ser., 334, 35

Silvotti, R., Schuh, S., Janulis, R., et al. 2007, Nature, 449, 189

Soker, N. 1998, AJ, 116, 1308

Southworth, J., Zucker, S., Maxted, P. F. L., \& Smalley, B. 2004, MNRAS, 355, 986

Vennes, S., Kawka, A., O’Toole, S. J., Németh, P., \& Burton, D. 2012, ApJ, 759, L25

Vučković, M., Aerts, C., Østensen, R., et al. 2007, A\&A, 471, 605

Vučković, M., Østensen, R., Bloemen, S., Decoster, I., \& Aerts, C. 2008, in Hot Subdwarf Stars and Related Objects, eds. U. Heber, C. S. Jeffery, \& R. Napiwotzki, ASP Conf. Ser., 392, 199

Wawrzyn, A. C., Barman, T. S., Günther, H. M., Hauschildt, P. H., \& Exter, K. M. 2009, A\&A, 505, 227

Webbink, R. F. 1984, ApJ, 277, 355

Wilson, R. E., \& Devinney, E. J. 1971, ApJ, 166, 605

Zorotovic, M., \& Schreiber, M. R. 2013, A\&A, 549, A95

Zorotovic, M., Schreiber, M. R., Gänsicke, B. T., \& Nebot Gómez-Morán, A. 2010, A\&A, 520, A86

\section{Appendix A: Emission lines from the secondary star}

Table A.1 gives a list of all emission lines from the secondary star of the AADor system we detected in the UVES spectra. Note that only the first 22 lines were used in the analysis.
Table A.1. List of emission features from the secondary.

\begin{tabular}{|c|c|c|c|}
\hline Ion & Wavelength $[\AA]$ & Peak & $E W[\mathrm{~m} \AA]$ \\
\hline $\mathrm{C}_{\text {II }}$ & 3920.68 & 0.01051 & -3.5 \\
\hline O II & 3954.37 & 0.00891 & -2.3 \\
\hline O II & 4069.75 & 0.01272 & -4.2 \\
\hline O II & 4072.16 & 0.01288 & -2.2 \\
\hline O II & 4075.87 & 0.01579 & -4.6 \\
\hline O II & 4089.29 & 0.00893 & -2.4 \\
\hline O II & 4119.22 & 0.01426 & -4.5 \\
\hline O II & 4153.30 & 0.00746 & -2.3 \\
\hline O II & 4189.79 & 0.00917 & -3.0 \\
\hline $\mathrm{C}_{\mathrm{II}}$ & 4267.10 & 0.02787 & -13.4 \\
\hline O II & 4414.91 & 0.02147 & -7.1 \\
\hline O II & 4416.97 & 0.01617 & -5.6 \\
\hline O II & 4590.97 & 0.01206 & -4.5 \\
\hline O II & 4596.17 & 0.00883 & -2.7 \\
\hline O II & 4641.81 & 0.01402 & -4.4 \\
\hline C III & 4647.40 & 0.01114 & -2.9 \\
\hline O II & 4649.14 & 0.01387 & -4.0 \\
\hline O II & 4661.64 & 0.00951 & -2.8 \\
\hline O II & 4676.23 & 0.00984 & -2.6 \\
\hline O II & 4699.21 & 0.00951 & -3.7 \\
\hline O II & 4705.35 & 0.01355 & -4.9 \\
\hline O II & 4943.06 & 0.01050 & -2.6 \\
\hline \multicolumn{4}{|c|}{ Lines detected, but not used in the analysis } \\
\hline He I & \multicolumn{3}{|c|}{$\begin{array}{l}(3819.61+3819.76),(3867.48+3867.63), 3871.82, \\
3935.91,3964.73,(4026.19+4026.36), \\
4120.81,4143.76,4168.97,4387.93,4471.69 \\
(4713.14+4713.37), 4921.929\end{array}$} \\
\hline Si III & \multicolumn{3}{|c|}{$3806.56,4552.65,4567.87$} \\
\hline Ca II & \multicolumn{3}{|l|}{3933.66} \\
\hline Mg II & \multicolumn{3}{|l|}{4481.13} \\
\hline N II & \multicolumn{3}{|c|}{$3994.99,3998.69,4035.09,4432.74,4447.03,4552.54$} \\
\hline C II & \multicolumn{3}{|c|}{$(3876.05+3876.19+3876.41), 3918.98$} \\
\hline C III & \multicolumn{3}{|l|}{$4647.40,4650.16$} \\
\hline O II & \multicolumn{3}{|c|}{$\begin{array}{l}3882.20,3919.29,3945.05,3973.26,3982.72,4035.09 \\
4060.58,4078.86,4085.12,4087.16, \\
4092.94,4097.26,4119.22,4132.81,4185.46,4192.50 \\
(4253.74+4253.98), 4275.52,4303.82, \\
4317.14,4319.63,4342.00,4342.83,4343.36,4344.42 \\
4345.56,4347.42,4349.43,4351.27,4353.60,4366.90 \\
4369.28,4378.41,4395.95,4448.21, \\
4452.38,4466.32,(4609.42+4610.14), \\
4638.85,4649.14,4650.84, \\
4696.36,(4701.23+4701.76), 4703.18, \\
4710.04,4906.88,4924.60,4941.12\end{array}$} \\
\hline
\end{tabular}

